\title{
A SIMPLE TRANSFER LEMMA FOR ALGEBRAIC SPECIFICATIONS
}

\section{J.A. BERGSTRA}

Department of Computer Science, University of Leiden, Wassenaarsweg 80, Postbus 9512, 2300 RA Leiden, Ti.e Netherlands

\author{
J.J.Ch. MEYER \\ Wiskundig Seminarium, De Boelelaan 1081, $1081 \mathrm{HV}$ Amsterdam, The Nethr.rlands
}

Received November 1980

Algebraic data types, initial algebra semantics, equational and conditional specifications

\section{Introduction}

Consider two data structures $\mathcal{V}$ and $\boldsymbol{w}$ modelled as algebraic structures $\left(\mathrm{D}_{\mathcal{v}} ; \Sigma_{\mathcal{v}}\right)$ and $\left(\mathrm{D}_{\mathcal{w}}: \Sigma_{i \nu}\right)$ where $\mathrm{D}_{\mathcal{v}}$ and $\mathrm{D}_{\mathcal{w}}$ are domains and $\Sigma_{\mathcal{v}}$ and $\Sigma_{\mathcal{w}}$ are signatures.

Let us assume that $\mathcal{V}$ and $\mathcal{W}$ have 'equally large' domains (i.e. \#( $\left.D_{\mathcal{V}}\right)=\#\left(D_{\mathcal{v}^{\prime}}\right)$ and \# stands for cardinality). Then we can compare their expressiveness by means of their signatures. We call $\mathcal{V}$ and $w$ equivalent with respect to expressiveness iff: if an element $\mathrm{v}$ in $\mathrm{D}_{\mathcal{V}}$ is represented by a $\Sigma_{\mathcal{V}}$-term, there is a $\Sigma_{\mathcal{W}}$ term that represents the element $w_{v}$ in $D_{w}$ corresponding to $v$, and conversely for an element $w$ in $D_{w}$ and its corresponding element $v_{w}$ in $D_{\mathcal{V}}$. (If we say that $\mathcal{V}$ and $\mathcal{W}$ are equivalent w.r.t. expressiveness, in future we shall always assume $\#\left(D_{\vartheta}\right)=\#\left(D_{w}\right)$, because it has no meaning otherwise.)

In this paper we shall prove the following theorem:

Theorem. Let $\mathcal{V}$ and $\mathcal{w}^{\mathcal{}}$ be two algebraic structures with equal expressiveness. Then it holds that: $\mathcal{V}$ has an initial algebra specification (in the sense of the ADJ Group) iff $\boldsymbol{w}$ has.

When proving this, which we shall do in section 2, we shall make explicit how a specification must be 'translated'.

In section 3 we shall illustrate this theorem by an exainple which in fact is an enrichment of the structure $\mathrm{SOI}_{\#}$ of our previous paper [2].

We assume that the reader is familiar with the basic work of the ADJ Group [3] but we shall refresh his memory by giving some exposition of the main notions in initial algebra specification in section 1, to which we shall now turn.

\section{Preparatory material}

Initial algebra semantics assigns to a specification $(\Sigma, E)$ in which $\Sigma$ is a signature (i.e. a set of symbols, each associated with a certain number $k$ of open places ( $k$ is called the arity of the symbol)), and $E$ is a set of equations over $\Sigma$, a unique meaning in the class ALG $(\Sigma, E)$ of all $\Sigma$-algebras satisfying ths equations of $E$ in the following way: two terms $t$ and $t^{\prime}$ over $\Sigma$ are identical iff $t$ and $t^{\prime}$ can be proved equal from the axioms in E (ses $[1,3]$; the semantics of the conditional equations are given in [5]).

An (n-sorted) algebra $\mathcal{V}$ of signature $\Sigma$ is a structure $\left(V_{1}, \ldots, V_{n} ; \Sigma\right)$ in which the $V_{1}$ are sets of ele. ments, called the domains of $\boldsymbol{V}$ and $\Sigma$ is a set of symbols naming functions $\sigma$ which are each defined on some cartesian product of the $V_{i}$ :

$\sigma: V_{\ell_{l}} \times \cdots \times V_{\ell_{k}} \rightarrow V_{m}$ 
where $1 \leqslant l_{1}, \ldots, l_{k}, m \leqslant n$, and naming special elements of the $V_{i}$, the so-called individual constants of $\mathcal{V}$, which can be considered as 0 -ary operators on $\mathcal{V}$. $\Sigma$ is called the signature of naming the constants of $\mathcal{V}$.

The following facts hold: let $\mathcal{V}$ and $\mathcal{W}$ be algebras of signature $\Sigma$ both finitely generated by their constants (i.e. $\mathcal{V}$ and $\mathcal{W}$ are minimal). Then: tive,

(1) any $\Sigma$-homomorphism $\phi: \mathcal{V} \rightarrow \mathcal{W}$ is subjec-

(i) if $\phi, \psi: \mathcal{V} \rightarrow \mathcal{W}$ are $\Sigma$-homomorphisms then $\phi=\dot{i}$,

(3) if there are $\Sigma$-homomorphisms $\phi: \mathcal{W} \rightarrow \mathcal{W}$ and $\psi: \mathcal{W} \rightarrow \mathcal{V}$, then $\mathcal{V} \cong \mathcal{W}$ (by either $\phi$ or $\psi$ ).

Let $\equiv$ be an equivalence relation on the n-sorted algebra $\mathcal{V}$; then we call a family of sets $J_{i} \subset V_{i}(1 \leqslant i \leqslant 1$.), such that $\forall b \in V_{i} \exists_{1} a \in J_{i}$ for which $b \equiv a$, a traversal for $\equiv$.

If $\Sigma$ is a signature, then $T(\Sigma)$ denotes the $\Sigma$-algebra of all terms over $\Sigma$ and $T_{\Sigma}\left[X_{1}, \ldots, X_{p}\right]$ denotes the algebra of polynomials in the indeterminates $X_{1}, \ldots, X_{p}$

If $\mathcal{V}$ is a $\Sigma$-algebra, then we mean by term evaluation in $\mathcal{V}$ a map val $v: T(\Sigma) \rightarrow \mathcal{V}$ with evaluates each term $t \in T(\Sigma)$ by substituting the constants of $\mathcal{V}$ by their names in $\mathrm{t}$. val $\vartheta$ can be defined uniquely as an epimorphism $\mathrm{T}(\Sigma) \rightarrow \mathcal{V}$.

If $\phi: \mathcal{V} \rightarrow \boldsymbol{W}$ is a homomorphism between $\Sigma$ algebras, then the following diagram commutes:

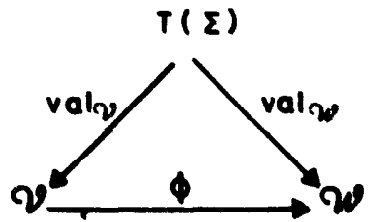

We define polynomial evaluation in $\mathcal{V}$ as the substitution of some terms $\vec{a}=\left(a_{1}, \ldots, a_{p}\right) \in(T(\Sigma))^{p}$ for indeterminates $\vec{X}=\left(X_{1}, \ldots, X_{p}\right)$ (where $a_{i}$ is a term naming an element of that domain over which $X_{i}$ ranges), followed by the evaluation of term $t(\vec{a})$ in $\mathcal{V}$.

An equation is a pair $\left(t(\vec{X}), t^{\prime}(\vec{X})\right)$ of polynomials from some $T_{\Sigma}\left[X_{1}, \ldots, X_{p}\right]\left(p>0 ;\right.$ if $p=0, T_{\Sigma}[\vec{X}]=$ $T(\Sigma))$ written as $t(\vec{X})=t^{\prime}(\vec{X})$, whereat it must be noted that $t(\vec{X})$ and $t^{\prime}(\vec{X})$ need not have any indeterminate in common.

A conditional equation is a formula of the form $M\left(t_{i}(\vec{X})=t_{i}^{\prime}(\vec{X})\right) \rightarrow t(\vec{X})=t^{\prime}(\vec{X})$.
If $E$ is a set of (conditional) equations over $\Sigma$ and $\mathcal{V}$ is a $\Sigma$-algebra such that $\mathcal{V} \vDash E$, we say that $\mathcal{V}$ is an $E$-algebra. We define $\operatorname{ALG}(\Sigma, E)$ as the class of all E-algebras and $\mathrm{T}(\Sigma, \mathrm{E})$ as the initial algebra for $\operatorname{ALG}(\Sigma, E)$, constructed from $\mathrm{T}(\Sigma) ; \mathrm{T}(\Sigma, E)=$ $T(\Sigma) / \equiv_{E}$ where $\equiv_{E}$ denotes the s.nallest congruence on $T(\Sigma)$ that identifies terms of $T(\Sigma)$ by means of the equations of $E$.

If $t \in T(\Sigma)$ we mean by $P_{E}(t)$ the $\equiv_{E}$-equivalence class $\in \mathbf{T}(\Sigma, E)$ that contains $t$.

An algebra $\mathcal{Y}$ of signature $\Sigma \boldsymbol{\nu}$ has a finite equational (conditional) specification $(\Sigma, \mathrm{E})$ if $\Sigma_{\nu}=\Sigma$, $E$ is a finite set of (conditional) equations over $\Sigma$, and $T(\Sigma, E) \cong \mathcal{V}$. More details can be found in $[1,3,5,6]$.

\section{Transferring specificaticins to equally expressible} data structures

In this section we shall reach the main goal of this paper. But in order to achieve this we must restate our definition of equivalency with respect to expressiveness from the introduction in a more formal way. (For notational convenience we only look at the single-sorted case.)

Definition. Two algebraic structures $\mathcal{V}=\left(\mathrm{D}_{\mathcal{V}} ; \Sigma_{\mathcal{V}}\right)$ and $\boldsymbol{w}=\left(\mathrm{D}_{w} ; \Sigma_{w}\right)$ are called equivalent with respect to expressiveness, notated as $\mathcal{} \approx \mathcal{\sim}$, iff

(i) there exists a bijective function $\phi: \mathrm{D}_{\vartheta} \rightarrow \mathrm{D}_{\mathfrak{w}}$,

(ii) for each element $f \in \Sigma_{\vartheta}$ there is a polynomial $t_{f}(\vec{X}) \in T_{\Sigma}\left[X_{1}, \ldots, X_{k}\right]$ (where $k \geqslant 0$ is the arity of $f ; k=0$ means that $f$ is an individual constant $c \in \Sigma_{v}$ ) such that the diagram

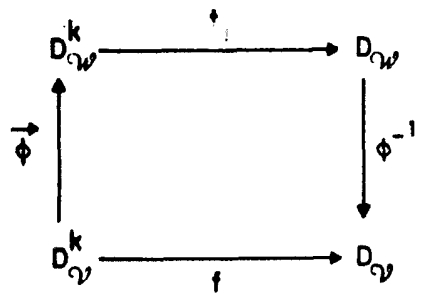

commutes, with

- $f$ is the k-ary operator on $D_{\mathcal{V}}^{k}$ named by $f$,

- $t_{f}$ is the $k$-ary operator on $D_{W}^{k}$, corresponding to the polynomial $t_{\mathrm{f}}(\vec{X}):\left(\forall \vec{X} \in D_{w}^{k}\right) t_{f}(\vec{x})=v l_{w} t_{f}(\vec{a})$ where $\vec{a}$ is a tuple of $k$ terms $\in T\left(\Sigma_{w}\right)$ such that 
$\operatorname{val}_{w}(\vec{a})=\vec{x}$, and

$\vec{\phi}\left(\mathrm{x}_{1}, \ldots, \mathrm{x}_{\mathrm{k}}\right)=\left(\phi\left(\mathrm{x}_{1}\right), \ldots, \phi\left(\mathrm{x}_{\mathrm{k}}\right)\right)$,

(iii) the same requirement as (ii) but with $\mathcal{V}$ and $\mathcal{w}$ interchanged, resulting in the commutative diagram

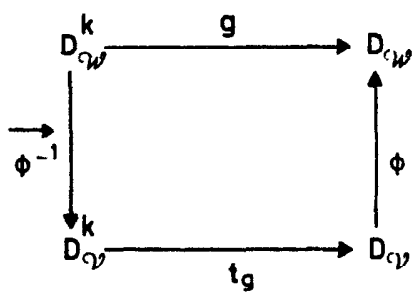

for a $g \in \Sigma_{w}$.

This definition is sufficiently formal to prove the

following theorem:

Theorem. If $\mathcal{\nu}=\left(\mathrm{D}_{\mathcal{v}} ; \Sigma_{\vartheta}\right)$ and $w=\left(\mathrm{D}_{w} ; \Sigma_{w}\right)$ are two algebraic structures such that $\mathcal{V} \approx \mathcal{W}$, then $\mathcal{W}$ has an initial algebra specification iff $\boldsymbol{w}$ has.

Proof. Suppose that $\mathcal{V}$ has a specification $\left(\Sigma_{\mathcal{V}}, \mathrm{E}_{\vartheta}\right)$ : $\mathcal{V} \cong \mathrm{T}\left(\Sigma_{\vartheta}, \mathrm{E}_{\vartheta}\right)$. Now we must prove that $\mathcal{W} \cong$ $\mathrm{T}\left(\Sigma_{w}, \mathrm{E}_{\mathcal{w}}\right)$ for some set $\mathrm{E}_{\mathcal{w}}$ of equations over $\Sigma_{* w}$. When constructing this $E_{x}$ we need the assumption that the operators named by the two signatures together, have been named consistently, i.e. two different operators must have different names. (If not so: rename them.)

As $\mathcal{V} \approx \mathcal{W}$ we have for each k-ary $f \in \Sigma \mathcal{V}(k \geqslant 0)$ a polynomial

$t_{f}(\vec{X}) \in T_{\Sigma_{w}}\left[X_{1}, \ldots, X_{k}\right]$

that satisfies (ii) of the previous definition. In the following we shall show that the set $\mathrm{E}_{\mathcal{v}}$ in which all $f \in \Sigma_{\mathcal{V}}$ have been replaced by their corresponding $t_{f}$, extended by the following equations: for each $g \in$ $\Sigma_{w}$ an equation

$g(\vec{X})=t_{g}^{\prime}(\vec{X})$

where $t_{g}^{\prime}(\vec{X})$ is $t_{g}(\vec{X})$ in which all elements $f \in \Sigma_{\nu}$ have been replaced by the corresponding $t_{\mathrm{f}} \in$ $\mathrm{T}_{\Sigma_{\mathcal{W}}}[\overrightarrow{\mathrm{X}}]$, satisfies the requirements to be $\mathrm{E}_{\mathcal{w}}$. First we define an enrichment $\mathcal{V}^{\prime}=\left(\mathrm{D}_{\mathcal{V}} ; \Sigma_{\mathcal{V}} \cup\right.$ $\Sigma_{\mathcal{W}}$ ) of $\mathcal{V}$, in which every (k-ary) $g \in \Sigma_{\mathcal{W}}$ (which names a function $\mathrm{g}: \mathrm{D}_{w}^{k} \rightarrow \mathrm{D}_{w}$ in the structure $w$ ) names a function $g_{\mathcal{V}}: D_{\mathcal{V}}^{k} \rightarrow D_{\mathcal{V}}$, such that the diagram

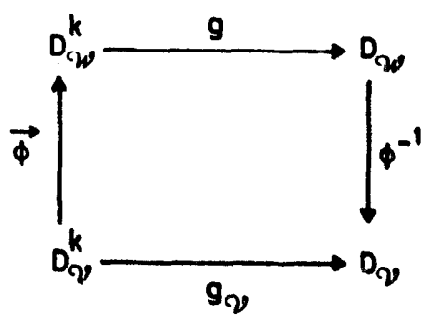

commutes.

Notice that it holds that $\forall_{\mathbf{g}} \in \Sigma_{\mathcal{Q}}$, naming $g$ in $w, \exists g_{\nu}$ such that $g_{v}\left(x_{1}, \ldots, x_{k}\right)=\phi^{-1} g\left(\phi\left(x_{1}\right), \ldots\right.$ $\left.\phi\left(x_{k}\right)\right)$ on one hand, and $\forall_{g} \in \Sigma_{w}$, naming $g$ in $w$, $\exists t_{g}$ such that $g\left(y_{1}, \ldots, y_{k}\right)=\phi t_{g}\left(\phi^{-1}\left(y_{1}\right), \ldots, \phi^{-1}\left(y_{k}\right)\right)$ on the other. So for $g v, t_{g}$ belonging to certain $g \in$ $\Sigma_{w}$ (naming $g$ in $w$ ) it holds that $g v\left(x_{1}, \ldots, x_{k}\right)=$ $\phi^{-1} \mathrm{~g}\left(\phi\left(x_{1}\right), \ldots, \phi\left(x_{k}\right)\right)=\phi^{-1} \phi \mathrm{t}_{\mathrm{g}}\left(\phi^{-1} \phi\left(x_{1}\right), \ldots\right.$ $\left.\phi^{-1} \phi\left(x_{k}\right)\right)=t_{g}\left(x_{1}, \ldots, x_{k}\right): g v(\vec{x})=t_{g}(\vec{x})$. But this means that $\left(\forall g \in \Sigma_{w}\right)$

$\mathcal{V}^{\prime} \vDash g\left(X_{1}, \ldots, X_{k}\right)=t_{g}\left(X_{1}, \ldots, X_{k}\right)$,

i.e. $\mathcal{V}^{\prime}$ is a homomorphic image of $\mathbf{T}\left(\Sigma_{\vartheta} \cup \Sigma_{W}\right.$, $\left.E_{\vartheta} \cup E^{*}\right)$ in which

$E^{*}=\left\{g(\vec{X})=t_{8}(\vec{X}) I_{g} \in \Sigma_{w}\right\}$.

(We shall abbreviate the equation $g(\vec{X})=t_{g}(\vec{X})$ (for certain $g \in \Sigma_{w}$ ) as $e_{g}$.) However, we also know that $\vartheta \cong \mathrm{T}\left(\Sigma_{\vartheta}, \mathrm{E}_{\vartheta}\right)$, which means that there is a traversal $J \subset T\left(\Sigma_{\vartheta}\right)$ for $\equiv_{E_{\nu}}$ and a function $\psi: D_{\nu} \rightarrow$ $U_{j \in J}\left\{e_{E_{\nu}}(j)\right\}$ such that $\psi$ is an isomorphism $\mathcal{\nu} \rightarrow$ $\mathbf{T}\left(\Sigma_{\nu}, \mathrm{E}_{\boldsymbol{\nu}}\right)$.

Clearly, the same $J$ is a traversal $\subset \mathbf{T}\left(\Sigma_{\nu} \cup \Sigma_{w}\right)$ for $\equiv_{E_{\mathcal{V}}} \cup E^{*}$ such that $\psi^{\prime}: D_{\mathcal{V}} \rightarrow U_{j \in J}\left\{e_{E_{\mathcal{V}} \cup E_{*}(j)}\right\}$ is an isomorphism $\mathcal{V}^{\prime} \rightarrow \mathbf{T}\left(\Sigma_{\mathcal{V}} \cup \Sigma_{\mathcal{W}}, \mathrm{E}_{\mathcal{V}} \cup \mathrm{E}^{+}\right)$, because $\forall t \in T\left(\Sigma_{v} \cup \Sigma_{w}\right) \Xi_{1} j \in J$ such that $t \equiv_{F W U} \cdot j$ by rewriting all $\Sigma_{W}$ symbols by means of the $e_{g} \in E^{*}$, and

(1) $\forall f \in \Sigma_{\mathcal{V}}$ it holds directly that

$f\left(\psi^{\prime}(x)\right) \equiv_{E} \cup E * \psi^{\prime}(f(x))$,

(2) $\forall g \in \Sigma_{w}:$

$g\left(\psi^{\prime}(x)\right) \stackrel{\left(o_{g}\right)}{\equiv} t_{g}\left(\psi^{\prime}(x)\right) \stackrel{(1)}{\equiv} \psi^{\prime}\left(t_{g}(x)\right)=\psi^{\prime}(g \psi(x))$

(where $\equiv$ is an abbreviation of $\equiv_{E_{\vartheta}} \cup E_{*}$ ). So, $\mathcal{V}^{\prime} \cong$ $\mathrm{T}\left(\Sigma_{\mathcal{V}} \cup \Sigma_{\mathcal{W}}, \mathrm{E}_{\mathcal{V}} \cup \mathrm{E}^{*}\right)$. 
Next we make an enrichment $\boldsymbol{W}^{\prime}$ of $\boldsymbol{W}$ in ar. analogous way:

$w^{\prime}=\left(\mathrm{D}_{\mathcal{W}} ; \Sigma_{\mathcal{V}} \cup \Sigma_{\mathcal{W}}\right)$.

Here we have that $\forall f \in \Sigma_{w}$ the diagram

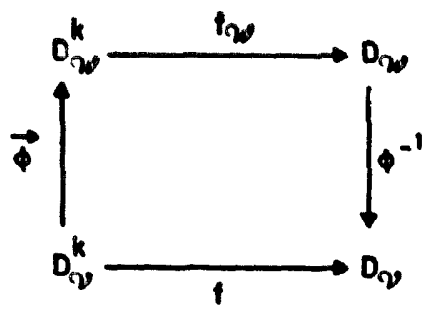

commutes, where $f$ is the function named by $f$ in $\mathcal{V}$, $f_{w}$ is the function named by $f$ in $W^{\prime}$ and $k$ is the

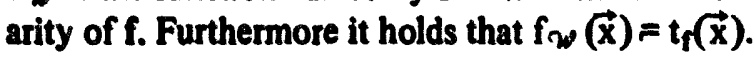

Lemma. $\phi$ is an isomorphism: $\mathcal{V}^{\prime} \rightarrow \mathcal{W}^{\prime}$.

Proof. $\phi$ was already a bijective function $D_{\vartheta} \rightarrow D_{w}$. Now it is also a homomorphism, because

$\forall f$ named by $\Sigma_{\mathcal{V}}: f_{\mathcal{w}}(\phi(x))=\phi(f(x))$,

Vgnamed by $\Sigma_{w:} g(\phi(x))=\phi(g v(x))$,

so for each $h \in \Sigma_{\boldsymbol{w}} \cup \Sigma_{\boldsymbol{w}}$ :

$\operatorname{val}(h(\phi(x)))=\operatorname{val}(\phi(h(x)))$.

\section{Corollary.}

$w^{\prime} \cong T\left(\Sigma_{\vartheta} \cup \Sigma_{w}, E_{\vartheta} \cup E^{*}\right)$.

Thus $\left(\Sigma_{\nu} \cup \Sigma_{w}, E_{\vartheta} \cup E^{*}\right)$ is a specification of $w^{\prime}$ too. As already $\omega^{\prime} \mid F E^{* *}$ with $E^{* *} \triangleq\left\{e_{\mathrm{f}}\right.$ |f $\left.\in \Sigma_{\vartheta}\right\}$ in which $e_{f}$ stands for $f(\vec{X})=t_{r}(\vec{X})$, we can also say:

$\boldsymbol{W}^{\prime} \cong \operatorname{T}\left(\Sigma_{\mathcal{V}} \cup \Sigma_{\boldsymbol{W}}, \mathrm{E}_{\mathcal{V}} \cup \mathrm{E}^{*} \cup \mathrm{E}^{* *}\right\}$.

But this in its turn is equivalent to:

$\boldsymbol{W}^{\prime} \cong T\left(\Sigma_{\mathcal{Y}} \cup \Sigma_{\mathcal{W}}, \mathbf{E}^{\prime}, \cup \mathbf{E}^{\prime} \cup \mathrm{E}^{* *}\right)$,

where $\mathrm{E}_{\mathcal{\nu}}^{\prime}$ and $\mathrm{E}^{\prime}$ are the sets $\mathrm{E}_{\mathcal{\nu}}$ and $\mathrm{E}^{\star}$ (respectively) of equations in which every $\Sigma_{\mathcal{V}}$-symbol $f$ has been replaced by te.

Next we look at the equations of $E^{* *}$. Notice that these all contain a $\Sigma_{\mathcal{V}}$-polynomial on the left side and a $\Sigma_{\boldsymbol{W}}$-polynomial on the right. As we have eliminated $\Sigma \boldsymbol{\nu}$-symbols out of all other equations (of $E_{\boldsymbol{\nu}}^{\prime}$ and $E^{\prime}$ ), it is intuitively clear that we can use an equation $e_{f} \in E^{* *}$ merely to abbreviate a $\Sigma_{w}$-term, but never to prove an identity between $\Sigma_{\mathcal{w}}$-terms, because in that case we must have expressed all terms in $\Sigma_{\mathscr{W}}$-symbols to match the equations $\in E_{\mathcal{\vartheta}}^{\prime} \cup E^{\prime}$. Formally we can prove this.

Lemma. For all $\Sigma_{w}$-terms $t_{1}, t_{2}$ :

$E_{v}^{\prime} \cup E^{\prime} \cup E^{* *} \vdash t_{1}=t_{2}, \quad$ iff $E_{v}^{\prime} \cup E^{\prime} \vdash t_{1}=t_{2}$.

Proof. The implication from the right side to the left is trivial: the fact that there are more equations implies that there are at least as many identities as before.

In order to prove the implication from the left to the right, it is sufficient by completeness to prove the following: $\forall t_{1}, t_{2} \in T\left(\Sigma_{w}\right)$ :

$\left(E_{\mathcal{V}}^{\prime} \cup E^{\prime} \cup E^{* *} k_{t_{1}}=t_{2}\right) \Rightarrow\left(E_{\mathcal{V}}^{\prime} \cup E^{\prime} k t_{1}=t_{2}\right)$ i.e. (for all structures $\mathcal{U}^{\prime} \in \operatorname{ALG}\left(\Sigma_{\nu} \cup \Sigma_{\mathcal{W}}\right)$ :

$\left(\mathcal{U}^{\prime} \vDash E_{\nu}^{\prime} \cup E^{\prime} \cup E^{* *}\right) \Rightarrow\left(\mathcal{U}^{\prime} \vDash \mathrm{t}_{1}=\mathrm{t}_{2}\right)$

implies for all structures $\mathscr{U} \in \operatorname{ALG}\left(\Sigma_{\boldsymbol{\nu}}\right)$ :

$\left(\mathcal{U} \vDash E_{\nu}^{\prime} \cup E^{\prime}\right) \Rightarrow\left(\mathcal{L} \vDash t_{1}=t_{2}\right)$.

Suppose $\mathcal{U}=\left(\mathrm{D}_{\mathcal{U}} ; \Sigma_{\mathcal{w}}\right) \in \operatorname{ALG}\left(\Sigma_{\mathcal{w}}, \mathrm{E}_{\mathcal{V}}^{\prime} \cup \mathrm{E}^{\prime}\right)$, arbitrarily chosen. Expand $\mathcal{U}$ to $\boldsymbol{U}^{\prime}=\left(\mathrm{D}_{\mathcal{U}}, \Sigma_{\mathcal{\vartheta}} \cup\right.$ $\left.\Sigma_{\mathscr{O}}\right)$ such that $\mathcal{U}^{\prime} \vDash E_{\mathcal{\nu}}^{\prime} \cup E^{\prime} \cup E^{* *}$. Then $\mathcal{U}^{\prime} \vDash$ $t_{1}=t_{2}$. But now also $\mathcal{U} \vDash t_{1}=t_{2}$, because $\mathcal{U}$ and $\mathcal{U}^{\prime}$ have the same domain.

This lemma yields the following corollary:

Corollary. If $\left.\mathcal{V}\right|_{\Sigma}$ stands for the $\Sigma$-reduct ${ }^{1}$ of $\mathcal{V}$, then

$$
\begin{aligned}
& \left.\mathrm{T}\left(\Sigma_{\mathcal{V}} \cup \Sigma_{\mathcal{W}}, \mathrm{E}_{\mathcal{V}}^{\prime} \cup \mathrm{E}^{\prime} \cup \mathrm{E}^{* *}\right)\right|_{\Sigma_{\mathcal{W}}}= \\
& \quad=\mathrm{T}\left(\Sigma_{\mathcal{W}}, \mathrm{E}_{\mathcal{V}}^{\prime} \cup \mathrm{E}^{\prime}\right) .
\end{aligned}
$$

Define $E_{\mathscr{\vartheta}} \triangleq E_{\mathcal{\vartheta}}^{\prime} \cup E^{\prime}$. Then

$$
\begin{aligned}
w & =\left.\left.w^{\prime}\right|_{\Sigma_{\mathcal{w}}} \cong \mathrm{T}\left(\Sigma_{\mathcal{V}} \cup \Sigma_{\mathcal{w}}, \mathrm{E}_{\mathcal{w}} \cup \mathrm{E}^{* *}\right)\right|_{\Sigma_{\mathcal{w}}} \\
& =\mathrm{T}\left(\Sigma_{w}, \mathrm{E}_{\mathcal{w}}\right) .
\end{aligned}
$$

\section{An application of the transfer lemma}

In [2] we already encountered the structure $\mathrm{SOI}_{\#}$ modelling finite sets of non-negative integers with

${ }^{1}$ The $\Sigma$-reduct of an algebra $\vartheta=\left(D_{\vartheta}: \Sigma_{\vartheta}\right)$ with $\Sigma \subset \Sigma_{\vartheta}$ is defined as the algebra $\left(\mathrm{D}_{\mathcal{V}} ; \Sigma\right)$. 
insertion. Now we look at enrichments of $\mathrm{SOI}_{\#}$ :

$\mathrm{SOI}_{1}=\left((\omega ; \mathrm{S},+, 0),(\mathrm{SETS} ; \boldsymbol{\phi}), \mathrm{IN}_{2} \#, \mathrm{DIFF}_{1}\right)$

and

$\mathrm{SOI}_{2}=\left((\omega ; \mathrm{S},+, 0),(\mathrm{SETS} ; \emptyset), \mathrm{IN}, \#, \mathrm{DIFF}_{2}\right)$

in which $\omega$ is the set of natural numbers,

$S$ names the successor $s$ on $\omega: s(x)=x+1$,

+ names the addition + on $\omega$,

0 names the constant $0 \in \omega$,

SETS is the class of finite sets of natural numbers,

names the empty set $\emptyset \in$ SETS

IN names the insertion operator in: $\omega \times$ SETS $\rightarrow$

SETS defined by in $(x, \xi)=\xi \cup\{x\}$,

\# names the operator \# : SETS $\rightarrow \omega$, giving of each

set $\in$ SETS its cardinality.

DIFF $_{1}$ names the operator diff 1 : SETS $\rightarrow$ SETS, defined by

$\operatorname{diff}_{1}\left(\xi_{1}, \xi_{2}\right)= \begin{cases}0 & \text { if } \xi_{1}=\xi_{2}, \\ 1 & \text { otherwise, }\end{cases}$

DIFF $_{2}$ names the operator diff $2:$ SETS $\rightarrow$ SETS, defined by

$\operatorname{diff}_{2}\left(\xi_{1}, \xi_{2}\right)= \begin{cases}1 & \text { if } \xi_{1}=\xi_{2}, \\ 0 & \text { otherwise. }\end{cases}$

First we note that $\mathrm{SOI}_{1} \approx \mathrm{SOI}_{2}:$ take for $\phi$ the identity ( $\omega \cup$ SETS $) \rightarrow(\omega \cup$ SETS $)$ and take

$t_{h}=h \quad$ for $h \in\{S,+, 0, \#, \phi, I N\}$,

$t_{r}\left(\Xi_{1}, \Xi_{2}\right)=\operatorname{DIFF}_{2}\left(\operatorname{IN}\left(\operatorname{DIFF}_{2}\left(\Xi_{1}, \Xi_{2}\right), \operatorname{IN}(0, \phi)\right)\right.$,

$$
\operatorname{IN}(0, \emptyset)) \text { for } f=\operatorname{DIFF}_{1} \text {, }
$$

and

$$
\begin{array}{r}
t_{g}\left(\Xi_{1}, \Xi_{2}\right)=\operatorname{DIFF}_{1}\left(\operatorname{IN}_{\left(D_{F} F_{1}\right.}\left(\Xi_{1}, \Xi_{2}\right), \operatorname{IN}(S(0), \emptyset)\right), \\
\operatorname{IN}(\operatorname{S}(0), \emptyset)) \quad \text { for } g=\operatorname{DIFF}_{2} .
\end{array}
$$

Although it is not at all clear how to specify $\mathrm{SOI}_{2}$ directly, we can now specify $\mathrm{SOI}_{1}$ first, which is a much easier thing to do, and then appiy our theorem.

Let $E_{1}$ be the set
(e $) \quad X+S(Y)=S(X+Y)$,
$\left(e_{2}\right) \quad X+0=X$,
(e $\left.e_{3}\right) \quad I N(X, I N(X, \Xi))=I N(X, \Xi)$,
(e $\left.e_{4}\right) \quad \mathbb{N}(X, I N(Y, \Xi))=\mathbb{I N}(Y, I N(X, \Xi))$,

(es) $\#(0)=0$,

$\left(e_{6}\right) \quad \#(\mathbb{I N}(X, \Xi))=\#(\Xi)+\operatorname{DIFF}_{1}(I N(X, \Xi), \Xi)$,

$\left(e_{7}\right) \quad \operatorname{DIFF}_{1}(\Xi, \Xi)=0$,

(e $\left.e_{8}\right) \operatorname{DIFF}_{1}\left(\Xi_{1}, \Xi_{2}\right)=\operatorname{DIFF}_{1}\left(\Xi_{2}, \Xi_{1}\right)$,

(eq) $\operatorname{DIFF}_{1}(\mathbb{I N}(X, \Xi), \rho)=S(0)$,

$\left(e_{10}\right) \operatorname{DIFF}_{1}(\mathrm{IN}(X+S(Y), \operatorname{IN}(X, \Xi)), \operatorname{IN}(X, \Xi))=$ $=\operatorname{DIFF}_{1}(\mathrm{IN}(\mathrm{X}+\mathrm{S}(\mathrm{X}), \Xi), \Xi)$,

$\left(e_{11}\right) \quad \operatorname{DIFF}_{1}\left(\operatorname{IN}\left(X, \Xi_{1}\right), \Xi_{2}\right)=\operatorname{DIFF}_{1}(\operatorname{IN}(0, \emptyset)$, IN $\left(0, \operatorname{IN}\left(D_{I F F}\left(\operatorname{IN}\left(X, \Xi_{2}\right), \Xi_{2}\right)\right.\right.$, IN(DIFF $\left.\left.\left.1\left(\mathbb{N}\left(X, \Sigma_{1}\right), \Sigma_{2}\right), 0\right)\right)\right)$ )

$\left(e_{12}\right) \operatorname{DIFF}_{1}(\operatorname{IN}(0,0), \operatorname{IN}(0, \operatorname{IN}(\mathrm{S}(0), \operatorname{IN}(X, 0))))=$ $=S(0)$.

and $\Sigma_{1}=\left\{S,+, 0,0, I N, \#, D_{1 F F}\right\}$.

Proposition. $\mathrm{SOI}_{1} \cong \mathrm{T}\left(\Sigma_{1}, \mathrm{E}_{1}\right)$.

Proof. Let

$$
\begin{aligned}
J_{1}= & \left\{S^{i}(0) \mid i \in \omega\right\}, \\
J_{2}= & \bigcup_{n \in 0}\left\{\mathbb{I N}\left(i_{1}^{n}, \mathbb{I N}\left(i_{2}^{n}, \ldots, \mathbb{I N}\left(i_{n}^{n}, \rho\right) \cdots\right)\right) \mid\right. \\
& \left.i_{1}^{n}<\cdots<i_{n}^{n} \in \omega\right\},
\end{aligned}
$$

where $n$ in $i_{k}^{n}$ is an upper index, and for $i \in \omega$, $i$ is short for $S^{i}(0)$. Then $x:$ SOI $_{1} \rightarrow T\left(\Sigma_{1}, E_{1}\right)$ defined by $\chi(\mathrm{z})=\left\{\begin{array}{l}\mathcal{E}_{\mathrm{E}}\left(\mathrm{S}^{2}(0)\right) \quad \text { if } \mathrm{z} \in \omega, \\ \varepsilon_{E}\left(\mathrm{IN}\left(\mathrm{r}_{1}(\mathrm{z}), \ldots, \mathrm{N}\left(\mathrm{r}_{\mathrm{m}}(\mathrm{z}), 0\right) \cdots\right)\right),\end{array}\right.$

if $z \in$ SETS and $r_{1}(z)<\cdots<r_{m}(z)$ are the elements of $z$, ordered according to magnitude with omission of equal elements, is an isomorphism: clearly $X$ is bijective, because by means of $E_{1}$ every SETS-sort term can be written uniquely in the form

IN $\left.\left(\mathbf{r}_{1}, \ldots, \mathbf{I N}\left(\mathbf{r}_{m}, \boldsymbol{\rho}\right) \cdots\right)\right)$

with $r_{1}<\cdots<r_{m} \in \omega$, and every w-sort term can be written uniquely as $S^{\prime}(0)$ for some $i \in \omega$. Moreover, $X$ is a homomorphism, because

(1) $s x(z) \equiv_{E_{1}} x(s(z))(\forall z \in \omega)$,

(2) $x\left(z_{1}\right)+x\left(z_{2}\right) \equiv_{E_{1}} x\left(z_{1}+z_{2}\right)\left(\forall z_{1}, z_{2} \in \omega\right)$,

(3) $I N(x(z), x(5)) \equiv_{E_{2}}$

$$
\begin{aligned}
& \left.\operatorname{IN}\left(z_{1}, \operatorname{IN}\left(r_{1}(\zeta), \ldots, r_{n}(\zeta), D\right) \cdots\right)\right) \equiv_{k_{1}} \\
& \left.\operatorname{IN}\left(r_{1}(\zeta \cup\{z\}), \ldots, r_{m}(\zeta \cup\{z\}, \emptyset) \cdots\right)\right)= \\
& =\phi(\zeta \cup\{z\})=\phi(\operatorname{in}(z, \zeta)) \\
& \quad \forall z \in \omega, \forall \zeta \in \operatorname{SETS} .
\end{aligned}
$$


(4) $\operatorname{DIFF}_{1}\left(x\left(\zeta_{1}\right), x\left(\zeta_{2}\right)\right) \equiv_{E_{1}} x\left(\operatorname{diff}_{1}\left(\zeta_{1}, \zeta_{2}\right)\right)$, because:

$x\left(\operatorname{diff},\left(\zeta_{1}, \zeta_{2}\right)\right)= \begin{cases}x(0)=0 & \text { if } \zeta_{1}=\zeta_{2}, \\ x(1)=s(0) & \text { if } \zeta_{1} \neq \zeta_{2},\end{cases}$

and

$\operatorname{DIFF}_{1}\left(x\left(\zeta_{1}\right), x\left(\zeta_{2}\right)\right){\Xi_{E_{1}}}_{1}$

$D_{I F F_{1}}\left(\mathrm{IN}\left(\mathrm{r}_{1}\left(\zeta_{1}\right), \ldots,\left(\mathrm{r}_{\mathrm{n}}\left(\zeta_{2}\right), 0\right) \cdots\right)\right.$,

$$
\left.\mathrm{IN}\left(\mathrm{r}_{1}\left(\zeta_{2}\right), \ldots,\left(\mathbf{r}_{m}\left(\zeta_{2}\right), \rho\right) \cdots\right)\right)
$$

$\left\{\begin{array}{l}\equiv_{\mathrm{E}_{1}} 0 \text { if } \zeta_{1}=\zeta_{2} \\ \text { if } \zeta_{1} \neq \zeta_{2}:\left(\exists_{i_{0}}\right) r_{i_{0}}\left(\zeta_{1}\right) \notin \zeta_{2} \vee\left(\exists_{j_{0}}\right) r_{j 0}\left(\zeta_{2}\right) \notin \zeta_{1},\end{array}\right.$

suppose the former case of these last two possibilities: then $(*)$ is

$$
\begin{gathered}
\equiv_{E_{1}} \operatorname{DIFF}_{1}\left(\mathbf { I N } \left(n_{0}\left(\zeta_{1}\right), \operatorname{NN}\left(r_{1}^{\prime}\left(\zeta_{1}\right), \ldots,\left(r_{n-1}^{\prime}\left(\zeta_{1}\right), \emptyset \cdots\right),\right.\right.\right. \\
\left.\operatorname{IN}\left(r_{1}\left(\zeta_{2}\right), \ldots,\left(r_{m}\left(\zeta_{2}\right), \rho\right) \cdots\right)\right) .
\end{gathered}
$$

Abbreviate this last term as $t_{1}$, then we can go on with (by $\left(e_{11}\right)$ )

$t_{1} \equiv_{E_{1}} \operatorname{DIFF}_{1}\left(\operatorname{IN}(0,0), \mathbb{I N}\left(0, \mathbb{N}\left(t_{2}, \operatorname{IN}\left(t_{1}, 0\right)\right)\right)\right)$

in which $t_{2} \triangleq$

$\operatorname{DIFF}_{1}\left(\mathrm{NN}\left(\mathrm{n}_{0}\left(\zeta_{1}\right), \mathrm{N}\left(\mathrm{r}_{1}\left(\zeta_{2}\right), \ldots,\left(\mathrm{r}_{\mathrm{m}}\left(\zeta_{2}\right), \emptyset\right) \cdots\right)\right.\right.$,

$$
\text { IN } \left.\left(r_{1}\left(\zeta_{2}\right), \ldots,\left(s_{m}\left(\zeta_{2}\right), 0\right) \cdots\right)\right) \text {. }
$$

Now $t_{2} \equiv_{E_{1}} S(0)$ by $\left(e_{10}\right)$ and $\left(e_{9}\right)$, so $t_{1} \equiv_{E_{1}} S(0)$ too by $\left(e_{12}\right)$.

(5) $\#(x(5)) \equiv E_{1} x(\#(\zeta))$, because:

$$
x(\#(\zeta))= \begin{cases}x(0)=0 & \text { if } \zeta=0, \\ x(m)=s^{m}(0) & \text { if } \zeta \text { contains exactly } \\ m(>0) \text { elements, }\end{cases}
$$

and

$H(x(s)) \equiv x_{1}$

$\left\{\begin{array}{l}\text { if } \zeta=0: \# x(\theta) \equiv \bar{x}_{1} \#(0) \equiv_{x_{1}} 0, \\ \text { if } \zeta \text { contains exactly } m(>0) \text { elements: }\end{array}\right.$

$$
\begin{aligned}
& \# \mathbb{N}\left(r_{1}(\zeta), \ldots,\left(r_{m}(\zeta), 0\right) \equiv_{E_{1}} \cdots \equiv_{E_{1}}\right. \\
& \equiv_{E_{1}} \#(0)+S(0)+\cdots+S(0) \equiv_{E_{1}} S^{m}(0)
\end{aligned}
$$

Remark. This proposition, by the way, means that the structure

$\mathrm{SOI}_{\#}=((\omega ; \mathrm{S}, 0),(\mathrm{SETS} ; 0), \mathrm{IN}, \#)$

of [2] has a hidden enrichment specification which fact was already mentioned in [4].

The application of our Theorem in the previous section now yields directly the following:

Corollary.

$\mathrm{SOI}_{2} \cong \mathrm{T}\left(\Sigma_{2}, \mathrm{E}_{2}\right)$

where

$\Sigma_{2}=\left\{S,+, 0,9, I N, \#, D_{1 F F}\right\}$

(naming the operators mentioned before) and $E_{2}$ is the set $E_{1}$ in which all occurrences of $\operatorname{DIFF}_{1}(\cdot$,$) have$ been replaced by

$\operatorname{DIFF}_{2}\left(\operatorname{IN}\left(\operatorname{DIFF}_{2}(\cdot ;), \operatorname{IN}(0, \emptyset)\right), \operatorname{IN}(0, \emptyset)\right)$,

united with the equation

(e) $\operatorname{DIFF}_{2}\left(\Xi_{1}, \Xi_{2}\right)=$

$$
\begin{aligned}
& =\text { DIFF }_{2}\left(\text { IN } _ { ( D _ { 1 F F } } \left(\operatorname { I N } _ { ( D _ { 1 F F } } \left(\operatorname { I N } \left(\operatorname{DIFF}_{2}\left(\Xi_{1}, \Xi_{2}\right)\right.\right.\right.\right. \text {, } \\
& \text { IN }(0, \varphi)), \operatorname{IN}(0, \varphi)), \operatorname{IN}(S(0), \emptyset)) \text {, } \\
& \mathrm{IN}(\mathrm{S}(\mathbf{0}),(\mathbf{)})), \mathrm{IN}(0,0)) \text {. }
\end{aligned}
$$

(We can omit the identities such as $S(X)=S(X)$ and =.) This example demonstrates how the transfer theorem can produce specifications of structures via specification of suitably chosen ones, which can be specified easily.

\section{References}

[1] J.A. Bergstra and J.V. Tucker, Algebraic specifications of computable and semicomputable data structures, Mathematical Centre, Department of Computer Science, Res. Rept. IW 115, Amsterdam (1979).

[2] J.A. Bergstra and J.J.Ch. Meyer, On specifying sets of integers, Institute of Applied Mathematics and Computer Science, University of Leiden, Report 80-21 (1980).

[3] J.A. Goguen, J.W. Thatcher and E.G. Wagner, An initial algebra approach to the specification, correctness and implementation of abstract data types, in: R.T. Yeh, Ed., Current Trends in Programming Methodology IV, Data Structuring (Prentice Hall, Engelwood Cliffs, NJ, 1978) 80-149.

[4] H.A. Klaeren, A simple class of algorithmic specifications for abstract software modules, Proceedings of MFCS'80, to appear.

[5] J.W. Thatcher, E.G. Wagner and J.B. Wright, Specification f abstract data types using conditional axioms, IBM T.J. Watsun Research Centre Report RC-6214, Yorktown Heights (1976).

[6] J.W. Thatcher, E.G. Wagner and J.B. Wright, Data tyr: specification: parameterization and the power of specifcation techniques, IBM T.J. Watson Research Center Report RC-7757, Yorktown Heights (1979). 